\title{
Uma investigação antropológica na terceira idade: concepções sobre a hipertensão arterial
}

\author{
An anthropological investigation of old age \\ and concepts concerning arterial hypertension
}

Fernanda Carval ho 1

Rodolpho Telarolli Junior 2

José Cândido Montei ro da Sil va Machado 3

1 Faculdade de Ciências e Letras de São José do Rio

Pardo. Av. Mario Ybarra de Almeida 1498, Araraquara, SP, 14800-420, Brasil.

2 Faculdade de Ciências

Farmacêuticas de

Araraquara, Universidade

Estadual Paulista.

Campus da Unesp, Rodovia

Araraquara-Jaú, Km 1,

Araraquara, SP

14801-902, Brasil.

3 Programa do Idoso,

Centro Regional de

Reabilitação de Araraquara,

Prefeitura do Município de

Araraquara, SP. Av. D. Pedro

II 1147, Araraquara, SP

14801-040, Brasil.
A bstract Anthropology and sociology are joining epidemiology, clinical investigation, and pathophysiology in studies on the aging process of the Brazilian population. The objectives of the present study were: a) to identify the concepts of the elderly population of the municipality of Araraquara, São Paulo, Brazil, towards the etiology of arterial hypertension $(\mathrm{AH})$ and the relevance of the different signs and symptoms that accompany the di sease; $b$ ) to improve el derly people's concepts towards the rel evance and utilizati on of different treatment categori es for $\mathrm{AH}$. Structured interviews were conducted with 29 individuals, the majority aged 60 years or over, who were being treated for hypertension at the Araraquara municipal gerontology outpatient clinic in August 1996. Patients were properly informed by the health team about problems related to $\mathrm{AH}$, as clearly perceived in the discourse of the elderly. Folkloric concepts pertaining to etiol ogy also appeared in the interviews and should betaken into consideration in the implementation of health education activities. Care provided by an interdisciplinary team was valued by the el derly. The most frequent complaint was the lack of free distribution of prescription medication for $\mathrm{AH}$ at the clinic.

Key words Hypertension; Anthropology; Aging Health

Resumo Essa pesquisa teve por objetivos: a) identificar as concepções da população idosa do muni cípio de Araraquara, SP, quanto à eti ologia da hi pertensão arterial ea rel evância dos diferentes sinais e sintomas que acompanham a doença; b) identificar as concepções dos idosos rel ativas à rel evância eà utilização das diferentes categorias de tratamento para HA. Foram realizadas entrevistas estruturadas com 29 indivíduos, a maioria com 60 anos ou mais, em tratamento de hipertensão em ambulatório de gerontologia da Prefeitura do Município de Araraquara, em agosto de 1996. Os paci entes encontram-se bem informados pela equipe de saúde sobre os problemas relaci onados à hi pertensão arterial. Concepções de etiologia tipicamente populares também apareceram, tratando-se de aspectos que devem ser consi derados quando da realização de ações de educação em saúde. $O$ atendimento por uma equipe multi disciplinar évalorizado pelos idosos, e a reclamação mais freqüente refere-se à falta dos medi camentos prescritos para entrega gratuita no ambulatório. Para mel horar a comuni cação entre equi pe de saúde e paci entes éimportantea inclusão de profissionais do campo das ciênci as humanas nas equi pes gerontológi cas. Palavras-chave Hipertensão; Antropologia; Saúde do Idoso 


\section{Introdução}

O objetivo da presente pesquisa foi o de identificar as concepções sobre hipertensão arterial entre pessoas idosas que se tratam no Centro Regional de Reabilitação de Araraquara, um serviço de saúde da Prefeitura do Município de Araraquara, São Paulo, a fim de se ter um diagnóstico acerca da importância que as representações sobre esse problema de saúde possam ter na busca e na manutenção de tratamento da doença.

Até pouco tempo atrás, o Brasil era considerado um país de jovens, e isso fez com que se desse pouca atenção aos idosos, particularmente por se tratar de um país com graves problemas sociais envolvendo crianças e jovens. Destacam-se, entre esses problemas, as deficiências nas áreas da saúde e educação, levando à tendência de não se considerar o problema do envelhecimento no rol das grandes questões sociais, sobretudo pela menor representatividade desse grupo na pirâmide etária populacional até os anos 70.

No último quarto de século, observou-se uma mudança significativa no padrão demográfico brasileiro, o que levou a caracterizar o Brasil como um "país jovem de cabelos brancos" (Veras, 1994). Caiu a fecundidade e a mortalidade, aumentando a longevidade da população e elevando a participação dos maiores de 60 anos no conjunto, faixa etária na qual se encontravam $7,2 \%$ dos brasileiros no censo de 1991 (FIBGE, 1991; Prata, 1994).

As estimativas sobre o ritmo do incremento da população são as mais variadas, havendo unanimidade em torno da previsão de que a população idosa crescerá mais rapidamente do que a população em geral. Uma estimativa razoável fala em quase 34 milhões de indivíduos com 60 anos e mais em 2025, fazendo do Brasil o sexto país do mundo com maior população idosa (Baeta, 1991). De 6,3\% da população total em 1980, as pessoas com 60 anos e mais passarão a 14\% em 2025 (Ramos et al., 1993).

Foi a partir da percepção desse rápido crescimento da proporção de idosos no Brasil que se tornaram mais comuns as pesquisas sobre a velhice no país. No caso específico da hipertensão arterial entre idosos no Brasil, há o recente trabalho de Lourenço (1996).

As pesquisas sobre as condições e necessidades dos idosos, no entanto, estão apenas começando, tanto no Brasil como na América Latina (OPS, 1992). Justifica-se, assim, a presente pesquisa, que pode contribuir para um melhor conhecimento das representações da doença entre pessoas idosas.
A hipertensão arterial como um problema de saúde pública

A hipertensão arterial vem se transformando progressivamente num dos mais graves problemas de saúde pública, atingindo adultos, em especial os mais idosos, sendo definida pela Organização Mundial de Saúde como a elevação crônica da pressão arterial sistólica e/ ou pressão arterial diastólica. Sua prevalência tende a ser maior no sexo masculino. Os números são muito variáveis para o país, variando de 5,0\% a 32,7\% em diferentes regiões do país (Lessa, 1993).

Um estudo realizado por Lolio (1990) em Araraquara, São Paulo, em 1987, revelou que nesse município as prevalências brutas de hipertensão arterial são superiores às de outras populações do Brasil e dos EUA, tendo encontrado $32,0 \%$ de hipertensos no sexo masculino e $25,3 \%$ entre as mulheres, com uma média de $28,3 \%$ para ambos os sexos, para os indivíduos entre 15 e 74 anos, moradores da zona urbana. No grupo de 65 a 74 anos de idade, a autora encontrou prevalências de 58,5\% e 54,9\%, respectivamente, para os homens e mulheres de Araraquara (Lolio et al., 1993). Neste município, Lolio (1990) encontrou a predominância das formas leves da doença, ao mesmo tempo que o consumo de medicamentos com ação terapêutica cardiovascular usados para doença hipertensiva era elevado. A mesma investigação mostrou que as mulheres tinham maior acesso aos serviços de saúde do que os homens. Uma de suas conclusões é a necessidade de se dar maior atenção a esse agravo por parte do sistema de saúde local, o que é muito importante, já que a hipertensão é um fator de risco para outras doenças, e do seu controle depende o declínio da mortalidade por doenças cerébrovasculares e coronariopatias.

Essas medidas tornam-se fundamentais para Araraquara, principalmente se considerarmos que a população idosa vem aumentando rapidamente nesse município. Pelos censos realizados em 1980 e 1991 vemos que a população com 60 anos e mais aumentou de $8,4 \%$ para $9,7 \%$, totalizando em números absolutos quase 16000 indivíduos (Telarolli Jr. et al., 1996). Isso coloca Araraquara com um índice de idosos superior à média do Estado de São Paulo. Como a hipertensão arterial é um problema que atinge de maneira especial os idosos, o aperfeiçoamento do controle da hipertensão junto a essa população passa a ser urgente.

Como objetivo geral da pesquisa, destacamos a identificação das concepções da população idosa quanto à etiologia da hipertensão ar- 
terial, bem como das concepções relativas à relevância dos diferentes sinais e sintomas que acompanham a hipertensão arterial. Foram também objetivos da investigação a identificação das concepções dos idosos relativas à relevância e à utilização das diferentes categorias de tratamento para hipertensão arterial (medicamentos, dieta e exercício físico), além de identificar aspectos da relação médico-paciente que sejam relevantes para o sucesso do tratamento da hipertensão arterial.

\section{Material e métodos}

A pesquisa foi realizada no Centro Regional de Reabilitação de Araraquara (CRRA), um serviço de saúde da Prefeitura do Município de Araraquara, São Paulo. A principal atividade econômica do município é a agro-indústria da canade-açúcar e da laranja, além da indústria metalúrgica e de processamento de alimentos. Araraquara contava com uma população de 171.687 habitantes, segundo estimativa da Fundação Seade para 1994.

Além de funcionar como referência em diversas áreas para o SUS na região de Araraquara, o CRRA mantém um programa de atendimento à população idosa do município. Esse programa conta com a participação de médico geriatra, clínico geral, psicólogo, enfermeiro, assistente social, fisioterapeutas, fonoaudiólogo e professor de educação física.

Foram entrevistados 29 pacientes dos dois sexos que já vinham sendo atendidos na instituição e que estavam sendo tratados de hipertensão arterial. As entrevistas foram realizadas por um dos investigadores (Carvalho). A entrevistas eram estruturadas, porém continham perguntas abertas. Também foram levantadas histórias de vida. As entrevistas foram gravadas, sendo realizadas em sala com isolamento acústico, ao longo dos mês de agosto de 1996. Não foram adotados outros procedimentos visando garantir a representatividade da amostra estudada para a população de idosos que se tratam no Centro Regional de Reabilitação de Araraquara. Tampouco foram adotados procedimentos de aleatorização na escolha dos entrevistados, além do balanceamento por sexo. Nessa pesquisa a utilização de métodos e técnicas não quantitativas não decorre de escolha ou preferência pessoal. São na verdade procedimentos necessários para se captar aspectos subjetivos da realidade social.

\section{Resultados e discussão}

Dos 10 homens entrevistados, 9 eram casados e apenas um solteiro. Entre as 19 mulheres 9 eram viúvas, 6 casadas, 3 solteiras e apenas 1 divorciada. Entre os homens, 7 eram aposentados, 1 trabalhava como jardineiro, 1 possuía uma pequena empresa e 1 não informou. Das 19 mulheres entrevistadas, 9 eram donas de casa, 1 era faxineira, outra era vendedora e uma terceira trabalhava cuidando de uma senhora idosa. Duas mulheres sempre trabalharam na lavoura, 2 eram empregadas domésticas, 1 era costureira e 2 eram comerciárias, uma das quais já se aposentara.

Em relação às causas presumíveis da doença, grande parte dos entrevistados referiu-se à hipertensão arterial como decorrência de estado emocional, traduzido como "passar muito nervoso". Um dos entrevistados apresentou uma causa peculiar, o fato de ter tomado um vinho licoroso, desencadeando a doença: “tomei um pouco de vinho licoroso, que eu não tomo nada, bebida alcoólica não é comigo, nem fumar, nem nada. Desde esse tempo nunca mais controlou a pressão...tomei um golinho só. Foi por isso que me deu problema de pressão". Trata-se de uma relação interessante, pois na etiologia popular é comum aparecerem causas “mágicas" para problemas de saúde. É dessa forma que interpretamos o discurso desse homem, creditando sua doença ao gole do vinho ingerido.

Alguns idosos relacionaram o início da doença a uma alimentação com muita gordura e sal. Isso provavelmente indica uma reformulação, por parte da população, do discurso médico, pois a dieta é muito recomendada pelos médicos como parte do tratamento. Também é importante notar que muitos dos entrevistados não responderam à pergunta sobre a causa da hipertensão, afirmando que não tinham idéia sobre o que poderia ter causado a doença. A concepção de causa mais valorizada, no entanto, é realmente a ligada ao stress da vida moderna - "passar muito nervoso".

A maioria dos entrevistados procurou algum serviço de saúde a partir do aparecimento dos sintomas mais freqüentes da hipertensão, como a dor de cabeça ou a sensação de "zoeira na cabeça". Dois dos entrevistados apenas iniciaram o tratamento depois da ocorrência de graves complicações associadas à hipertensão arterial - um teve acidente vascular cerebral e outro um infarto agudo do miocárdio. A sensação de cansaço foi outro sintoma muito valorizado. Alguns se referiram à canseira e "fadigação" como o motivo que os levou a procurar um 
médico. Tontura e náusea são outros sintomas que foram valorizados por parte dos entrevistados. Poucos entrevistados se referiram aos perigos da pressão al ta, e os que falaram apenas reproduziram o discurso médico, uma vez que tinham obtido informações a respeito dos próprios médicos.

Pode-se notar pelas entrevistas que a necessidade do tratamento não é, em grande parte, compreendida. Isso provavelmente se deve ao fato de se tratar de uma doença crônica que, quando controlada com o uso de medicamentos, pode permanecer sem sintomas por longo tempo. Uma conseqüência indireta desse fato é que, em decorrência da capacidade de eliminar os sintomas, alguns pacientes acabam ingerindo o medicamento apenas quando sentem os sintomas que os incomodam, aumentando o risco de ocorrência de complicações cárdio-circulatórias. Como observou Lefèvre (1990) a respeito de pacientes hipertensos, "sendo a doença uma sensação, o paciente não se sente doente. Mas a despeito de não se sentir (para usar o pleonasmo) internamentedoente, ele o é externamente" (p.155). Em outras palavras, com a ausência de sintomas o paciente não se sente doente, mas ele sabe de sua condição pelos médicos que o tratam, ou seja, externamente.

A presença de sintomas éo que faz com que os indivíduos atribuam a si mesmos a capacidade de administrar os medicamentos, em função dos sinais e sintomas por eles atribuídos à hipertensão. Isso apareceu com grande clareza nos depoimentos de dois entrevistados, que deixam de tomar sua prescrição al gumas vezes durante dois ou três dias, desde que se sintam bem.

É consenso entre os geriatras a ocorrência freqüente de múltiplos distúrbios de saúde num mesmo paciente idoso (Moriguchi \& Moriguchi, 1988; Nascimento et al., 1989; Cançado, 1994; Carvalho-Filho \& Papaléo-Netto, 1994). Padrão semelhante também foi encontrado entre as pessoas entrevistadas, e algumas não davam muita importância à hipertensão, valorizando mais os outros problemas de saúde. Isto se explica pelo fato de que tomavam medicamentos para vários outros problemas (diabetes, "para os rins", "para varizes" e "para o cérebro"). Este fato certamente dificulta o reconhecimento dos efeitos colaterais relativos a cada um dos medicamentos ingeridos.

Mesmo assim, vários entrevistados reclamaram dos efeitos colaterais atribuídos à medicação para hipertensão arterial. Uma das muIheres, por exemplo, relatou o fato de que o Capoten Ihe provocava muito inchaço na perna e que, após sua queixa, o médico substituiu a medicação por Lopril, com o qual ela se deu bem. Na visão da paciente isso acontece porque o segundo remédio "tem menos mistura". É interessante notar que o Lopril contém a mesma droga que o Capoten, o captopril, associada a um produto diurético, a hidroclorotiazida. Levando isso em conta, na verdade é o segundo medicamento prescrito para a paciente que contém 'mais mistura'. Outra senhora se queixou que, com o uso continuado de Aldomet, ficava completamente “Iargada na cama”, acrescentando que "o Aldomet tem muita mistura e prejudica o organismo".

Existe também um preconceito quase generalizado contra o fato de se usar regularmente medicamentos. Foram comuns afirmações como "eu não gosto muito de tomar remédio" ou " tomar demais faz mal". Essa forma de sentir a medicalização é especialmente prejudicial para o sucesso terapêutico quando se trata de uma doença crônica como a hipertensão arterial. Outro motivo preponderante para o desgosto com o uso do remédio é o seu alto preço. Pudemos constatar, ainda, um certo preconceito dos idosos em relação ao tratamento alopático. Isso ficou claro na afirmação de um senhor: "tem muita mistura, muita química que faz mal para o organismo". Alguns entrevistados fizeram referência explícita ao uso alternativo de produtos naturais, como chá de folhas de eucalipto sem açúcar ("afina o sangue, emagrece e a pressão baixa"). Quando abordamos os tratamentos medicamentosos em seu conjunto, encontramos alguns padrões de atitudes que são comuns frente ao controle da hipertensão arterial : a maioria dos idosos entrevistados encontrava-se consciente da necessidade do tratamento; alguns não gostam de utilizar remédios por preconceito contra os medicamentos alopáticos; os que não usam regularmente os remédios prescritos consideram normal a pressão subir quando estão nervosos. $\mathrm{O}$ seguinte padrão de comportamento frente ao uso de medicamentos foi observado: 22 entrevistados usavam o medicamento constantemente; 5 não estavam usando nenhum produto, sendo que 2 haviam recebido prescrição médica, mas abandonaram o tratamento; 2 tomam sua prescrição somente quando percebem os sintomas mais valorizados, como dor de cabeça, tontura e canseira, a despeito de orientação em contrário. Segundo informação de um dos geriatras do CRRA, alguns pacientes deixam de tomar a medicação para "testar se o remédio está fazendo efeito". A dieta é muito valorizada e quase todos seguem a dieta recomendada pelo médico, evitando sal e comida 
gordurosa. Parte dos idosos também estavam fazendo dieta para perder peso, conscientes do problema da obesidade como um fator de risco para a hipertensão arterial.

Outro aspecto do tratamento que foi bastante enfatizado pelos entrevistados é a necessidade de exercício físico como coadjuvante no controle da hipertensão arterial. A caminhada é a modalidade de exercício mais realizada entre os idosos, e a ginástica, realizada nas instalações do CRRA é também encarada como uma atividade de lazer, que Ihes proporciona uma oportunidade de convívio social.

Pela análise das entrevistas pudemos constatar que os idosos atendidos pelo Centro Regional de Reabilitação de Araraquara estão bem informados a respeito da necessidade de tratamento da hipertensão arterial. Reinterpretações com base no discurso médico são freqüentes em relação à etiologia e tratamento da doença.

\section{Referências}

BAETA, A. M. C., 1991. Transição demográfica e novas demandas em saúde: 0 atendimento à terceira idade. Revista deAdministração Pública, 25:17378.

CANCCADO, F. A. X., 1994. Noções Práticas de Geriatria. Belo Horizonte: Coopmed \& Health C. R.

CARVALHO FILHO, E. T. \& PAPALÉO NETTO, M., 1994. Geriatria - Fundamentos, Clínica eTerapêutica. São Paulo: Livraria Atheneu Editora.

FIBGE (Fundação Instituto Brasileiro de Geografia e Estatística), 1991. Censo Demográfico do Brasil 1991. Rio de Janeiro: Fundação IBGE.

LEFÈVRE, F., 1990. O Medicamento como Problema de Saúde Pública. Contribuição para o Estudo de uma Mercadoria Simbólica. Tese de Doutorado, São Paulo: Faculdade de Saúde Pública, Universidade de São Paulo.

LESSA, I., 1993. Estudos brasileiros sobre a epidemiologia da hipertensão arterial: análise crítica dos estudos de prevalência. Informe Epidemiológico do SUS, 3:59-75.

LOLIO, C. A., 1990. Prevalência da hipertensão arterial em Araraquara. Arquivos Brasileiro de Cardiologia, 55:167-173.

LOLIO, C. A., PEREIRA, J. C. R.; LOTUFO, P. A. \& SOUZA, J. M. P., 1993. Hipertensão arterial e possíveis fatores de risco. Revista de Saúde Pública, 27:357-362.
A pouca informação sobre as causas da hipertensão arterial pode refletir o fato de ser essa uma doença de natureza multifatorial, assim que os próprios médicos têm dificuldade em estabelecer uma causa unívoca para cada paciente.

O serviço prestado pelo CRRA foi valorizado pelos entrevistados, sobretudo o fato de se tratar de um serviço em que eles dispõem de uma equipe multiprofissional para atendê-los.

O sucesso de programas de saúde voltados à população idosa, em especial os de controle da hipertensão arterial, deve, necessariamente, considerar as representações do grupo sobre a doença, com destaque para suas concepções, considerando os sinais e sintomas mais freqüentes. Não é demais lembrar que a equipe de saúde deve ter sempre em mente que os indivíduos, e os idosos não são exceção, reelaboram e reinterpretam o discurso médico, traduzindo-o para o seu universo.

LOURENÇO, R. A., 1996. Hi pertensão Arterial em uma População de Idosos. Dissertação de Mestrado, Rio de Janeiro: Instituto de Medicina Social, Universidade do Estado do Rio de Janeiro.

MORIGUCHI, Y. \& MORIGUCHI, E. H., 1988. Biologia Geriátrica Ilustrada. São Paulo: Fundo Editorial BIK.

NASCIMENTO, E. C.; HAMADA, G. S. \& MORIGUCHI, Y., 1989. Características das doenças no idoso. Revista de Medicina da PUC/RS, 7:170-174.

OPS (Organización Panamericana de la Salud), 1992. La Salud de los Ancianos: una Preocupación de Todos. Washington: OPS.

PRATA, P. R., 1994. Desenvolvimento econômico, desigualdade e saúde. Cadernos de Saúde Pública, 10:387-391.

RAMOS, L. R.; ROSA, T. E.; OLIVEIRA, Z. M.; MEDINA, M. C. G. \& SANTOS, F. R. G., 1993. Perfil do idoso em área metropolitana na região sudeste do Brasil: resultados de inquérito domiciliar. Revista de Saúde Pública, 27:87-94.

TELAROLLI Jr., R.; MACHADO, C. M. S. \& CARVALHO, F., 1996. Perfil demográfico e condições sanitárias dos idosos em área urbana do Sudeste do Brasil. Revista de Saúde Pública, 30:485-498.

VERAS, R. P., 1994. PaísJovem Com Cabelos Brancos: A Saúde Do Idoso no Brasil. Rio de Janeiro: Relume Dumará. 